\section{Low-distortion switched-capacitor event-driven analogue to-digital converter}

\section{Majidzadeh, A. Schmid and Y. Leblebici}

An event-driven tracking analogue-to-digital converter (ADC) architecture is proposed. The proposed architecture has less sensitivity to amplifier and DAC nonlinearity, and reduces the swing and dynamic common-mode range requirement of the operational transconductance amplifier and comparators, respectively. The efficiency of the ADC is confirmed by detailed circuit simulations.

Introduction: Emerging ultra-low-power applications such as wireless sensor networks and wireless body area networks require the integration of very efficient signal processing techniques, specifically in analogueto-digital conversion. General-purpose ADCs that rely on Nyquist theory result in unnecessary sampling, and dissipate excessive power within the ADC and subsequent digital signal processing units during periods of low activity. Activity dependent [1], variable resolution [2] ADCs, and continuous time digital signal processing [3] are the most recent techniques to tackle the aforementioned issues. This Letter presents an event-driven ADC architecture, which enables a significant relaxation of the analogue circuit specifications and improvement of the performance.

Event-driven converters: The architecture of an event-driven converter is shown in Fig. 1 [1]. The input signal is scaled-up through a lownoise driver amplifier to the ADC input dynamic range, and is compared with discrete outputs of the resistor string DAC (RSDAC). At each levelcrossing event, the output of the RSDAC is increased or decreased by a voltage step of $V_{\mathrm{LSB}}$ equivalent to one least significant bit (LSB) of the $\mathrm{ADC}$, always keeping the input signal within a voltage interval defined by the RSDAC outputs $V_{\text {up }}$ and $V_{\text {down }}$ and which has a magnitude equal to one $V_{\mathrm{LSB}}$. Thus, the tracking accuracy is defined by the RSDAC resolution. Since sampling is not employed in the conversion process, the architecture is very robust to out-of-band interferers, which mitigates the need for an aggressive anti-aliasing filtering and helps to reduce the system total power consumption. However, the circuit implementation of this architecture suffers from several drawbacks. Since the input signal is processed by a low-noise amplifier (LNA), a linear and large output swing LNA is required, which is not straightforward to design in lowvoltage regimes. Moreover, rail-to-rail input-stage comparators are necessary to handle the large amplitude signals (large common-mode variation) at the comparator inputs. Finally, the architecture is susceptible to the mismatch of the unit elements of the RSDAC, which may cause in-band harmonic distortion.

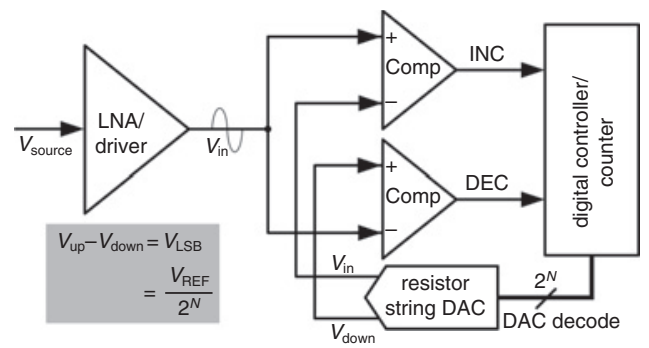

Fig. 1 Conventional event-driven converter

Low-distortion event-driven converter: The proposed architecture is shown in Fig. 2. The digital controller and RSDAC of Fig. 1 are replaced with an up/down analogue counter, a switched-capacitor (SC) integrator, and the feedback signal is referred to the input of the LNA. Thus, the LNA processes the tracking error, i.e. quantisation noise, instead of the input signal. Consequently, the signal swing at the output of the LNA is dramatically reduced and is limited to one $V_{\mathrm{LSB}}$ in a single-ended design, relaxing the linearity requirement of the LNA [4]. Moreover, the slew-rate requirement of the LNA is considerably mitigated owing to the reduced voltage swing. The integrator, subtractor and LNA enclosed in the dashed box are implemented using a single OTA, i.e. without any additional active element. Since subtraction is performed at the input of the LNA instead of the inputs of the comparators, the comparison is conducted with respect to fixed reference voltages, i.e. not with respect to the dynamic outputs of the RSDAC. Removing the dynamic common-mode voltage at the input of the comparators, rail-to-rail input-stage comparators are not required, which helps to reduce the power consumption. The nonlinearity issue of the multi-bit RSDAC is resolved by using a single-bit SC integrator, which is controlled by clock phases generated by comparator output increment (INC) and decrement (DEC) signals. A continuous-in-time and discrete-in-amplitude output is obtained using an asynchronous Gray-code up/down counter, which also minimises any potential error in consecutive synchronous sampling for spectral analysis purposes.

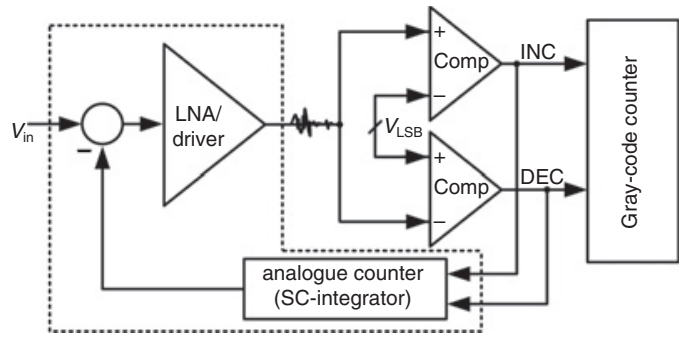

Fig. 2 Proposed event-driven converter

Circuit implementation: The circuit implementation of the proposed architecture and its corresponding timing diagram covering several consecutive crossing events is shown in Fig. 3. A fully differential realisation is employed, but a single-ended view is shown for the sake of simplicity. The continuous-time signal path formed by $\mathrm{C}_{1}$ and $\mathrm{C}_{2}$ provides additional gain for the input signal. The subthreshold diodeconnected transistors in the feedback path provide the input commonmode voltage of the LNA, in long periods of signal inactivity. The integrator is realised by means of two time-interleaved capacitors $C_{2}$, which are controlled by non-overlapping phases of $\varphi_{1}$ and $\varphi_{2}$ as a way to relax the bandwidth requirement of the LNA, and at the cost of a negligible penalty related to the mismatch between two capacitors. It can be shown that the residue of the error at the LNA output, which is due to the mismatch of the two capacitors, integrates to zero for two consecutive INC or DEC crossing events, and is bounded to a maximum of one $V_{\mathrm{LSB}}$ for consecutive INC and DEC events, which occurs at zero input signal. The resolution of the ADC is adjusted by tuning parameter $k$ or $V_{\mathrm{LSB}}$. Differential comparators detect the crossing events. The DEC and INC signals are generated using the same differential reference voltages as in the integrator. Comparator offsets are cancelled by isolated DC operating points provided by highpass filtering in front of the comparators [1]. At each crossing event, the DFF generates an integration phase which is valid until the next crossing event in order to utilise the entire timing slot between two consecutive events for linear settling of the integrator. A conventional non-overlaping phase generator is used to generate non-overlapping integration phases $\varphi_{1}$ and $\varphi_{2}$ and the corresponding up/down integration phases $\varphi_{1 \mathrm{u}, \mathrm{d}}$, and $\varphi_{2 \mathrm{u}, \mathrm{d}}$, respectively.

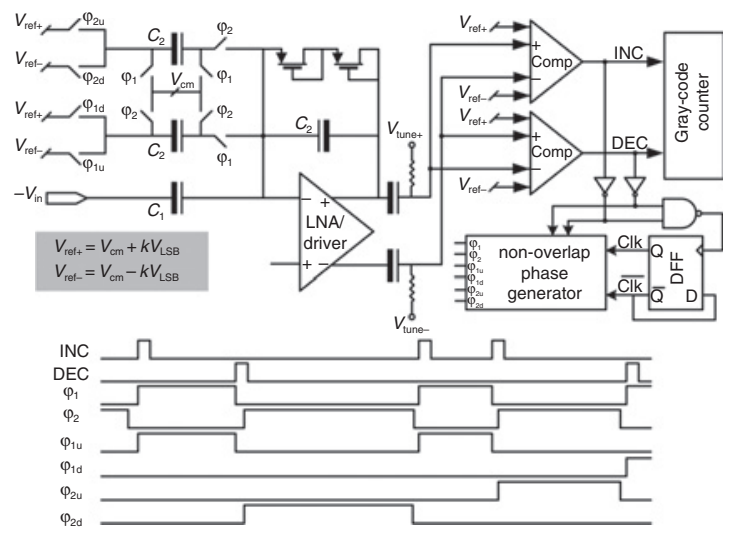

Fig. 3 Circuit implementation and corresponding timing diagram of proposed architecture

Simulation results: The proposed structure has been simulated using a $0.18 \mu \mathrm{m}$ CMOS technology with a $1.8 \mathrm{~V}$ supply voltage. The ADC can handle a full scale of $1 \mathrm{~V}_{\mathrm{p}-\mathrm{p}}(0 \mathrm{dBFS})$ single-tone input signal up 
to a frequency of $1.8 \mathrm{kHz}$ without slope overloading and a total static current consumption of $6.8 \mu \mathrm{A}$. The higher input frequencies are processed with smaller amplitudes, e.g. $-6 \mathrm{dBFS}$ at $3.9 \mathrm{kHz}$. The output spectrum and dynamic range (DR) plot of the 8-bit ADC using an OTA with $31 \mathrm{~dB}$ of DC gain is shown in Fig. 4. Synchronous sampling at $250 \mathrm{kHz}$ at the output of the Gray-code counter is used for spectral analysis purposes. The spurious free dynamic range (SFDR) with $0 \mathrm{dBFS}$ input is simulated at $52.1 \mathrm{~dB}$ without considering any mismatch between the time-interleaved capacitors $\mathrm{C}_{2}$, and slightly degrades to $50.4 \mathrm{~dB}$ by introducing the $3 \%$ of mismatch. The signal-to-quantisation-noise ratio (SQNR) integrated up to a frequency of $15.625 \mathrm{kHz}$ is 46.8 and $45.8 \mathrm{~dB}$ without and with $\mathrm{C}_{2}$ mismatch, respectively. The DR of the ADC is simulated at $48.6 \mathrm{~dB}$. Better SQNR performance may be achieved by continuous-time filtering-out-of band components prior to synchronous sampling.

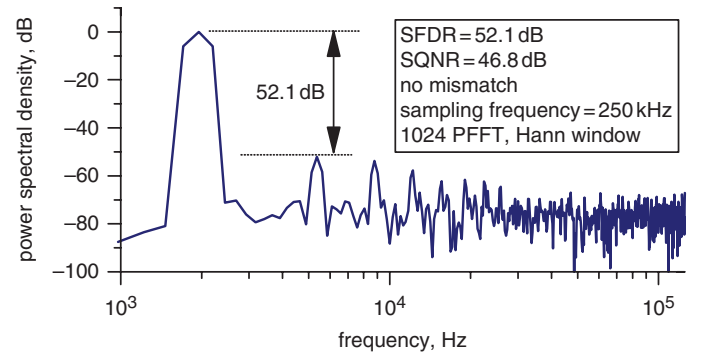

a

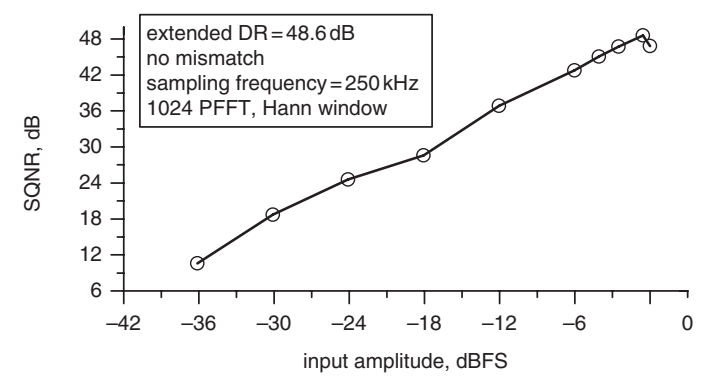

$b$

Fig. 4 Output spectrum of $A D C$ and $D R$ of $A D C$ after synchronous sampling $a$ Output spectrum of ADC $b$ Dynamic range of ADC
Conclusion: A clock-less tracking low-distortion ADC is presented. Simulation results of the ADC prove the robustness of the architecture with respect to analogue circuit non-idealities, at low static power dissipation.

(C) The Institution of Engineering and Technology 2010 30 June 2010

doi: $10.1049 / \mathrm{el} .2010 .1785$

One or more of the Figures in this Letter are available in colour online.

V. Majidzadeh, A. Schmid and Y. Leblebici (Institute of Electrical Engineering, Ecole Polytechnique Fédérale de Lausanne, 1015 Lausanne, Switzerland)

E-mail: vahid.majidzadeh@epfl.ch

\section{References}

1 Schell, B., and Tsividis, Y.: 'A continuous-time ADC/DSP/DAC system with no clock and with activity-dependent power dissipation', IEEE J. Solid-State Circuits, 2008, 43, (11), pp. 2472-2481

2 Kurchuk, M., and Tsividis, Y.: 'Signal-dependent variable-resolution clockless A/D conversion with application to continuous-time digital signal processing', IEEE Trans. Circuits Syst. I, 2010, 57, (5), pp. $982-991$

3 Tsividis, Y.: 'Continuous-time digital signal processing', Electron. Lett., 2003, 39, (21), pp. 1551-1552

4 Silva, J., Moon, U., Steensgaard, J., and Temes, G.C.: 'Wideband lowdistortion delta-sigma ADC topology', Electron. Lett., 2001, 37, (12), pp. $737-738$ 